PROCEEDINGS OF THE

AMERICAN MATHEMATICAL SOCIETY

Volume 127, Number 12, Pages 3547-3556

S 0002-9939(99)04918-7

Article electronically published on May 13, 1999

\title{
LACUNARY SETS BASED ON LORENTZ SPACES
}

\author{
RAYMOND J. GRINNELL
}

(Communicated by J. Marshall Ash)

\begin{abstract}
A new lacunary set for compact abelian groups is introduced; this is called a $\Lambda(p, q)$ set. This set is defined in terms of the Lorentz spaces and is shown to be a generalization of $\Lambda(p)$ sets and Sidon sets. A number of functional-analytic statements about $\Lambda(p, q)$ sets are established by making use of the structural similarities between $L^{p}$ spaces and Lorentz spaces. These statements are analogous to several well-known properties of a set which are equivalent to the definition of a $\Lambda(p)$ set. Some general set-theoretic and arithmetic properties of $\Lambda(p, q)$ sets are also developed; these properties extend known results on the structure of $\Lambda(p)$ sets. Open problems and directions for further research are outlined.
\end{abstract}

\section{INTRODUCTION}

Throughout this paper $G$ denotes an infinite compact abelian group and $\Gamma$ its discrete dual group. If $X \subseteq L^{1}$ and $E \subseteq \Gamma$, let $X_{E}=\{f \in X: \hat{f}(\gamma)=0$ for all $\gamma \notin E\}$. For $p \in(1, \infty)$ recall that a subset $E$ of $\Gamma$ is called a $\Lambda(p)$ set if $L_{E}^{p}=L_{E}^{1}$. The set $E$ is called a Sidon set if $L_{E}^{\infty} \subseteq\left\{f \in L^{1}: \hat{f} \in \ell^{1}(\Gamma)\right\}$. Sidon sets and $\Lambda(p)$ sets are the most widely studied types of lacunary sets for compact abelian groups; two standard references on the theory of these sets are [10] and [12]. In this paper we introduce and study a new type of lacunary set which is defined in terms of the Lorentz spaces, $L(p, q)$.

Definition 1.1. Let $p \in(1, \infty)$ and $q \in[1, \infty)$. A subset $E$ of $\Gamma$ is called a $\Lambda(p, q)$ set if $L(p, q)_{E}=L_{E}^{1}$.

What motivates the notation for $\Lambda(p, q)$ sets is the notation for Lorentz spaces. These spaces are a two-parameter family of function spaces which are closely related to the $L^{p}$ spaces. In particular, they are intermediate to the $L^{p}$ spaces in the sense that whenever $1 \leq q<p<r \leq \infty$,

$$
L^{\infty} \subset \bigcup_{t>p} L^{t} \subseteq L(p, q) \subset L^{p} \subset L(p, r) \subseteq \bigcap_{s<p} L^{s} \subset L^{1} .
$$

Furthermore, each $L^{p}$ space is itself a Lorentz space as $L^{p}=L(p, p)$. It follows from this that every $\Lambda(p)$ set is also a $\Lambda(p, p)$ set. In [6, Section 3] two types of lacunary sets based on Lorentz spaces are introduced; these are called $\Lambda_{1}(p, q)$ and $\Lambda_{2}(p, q)$ sets. We shall see that these sets are also examples of $\Lambda(p, q)$ sets.

Received by the editors September 5, 1996 and, in revised form, February 12, 1998.

1991 Mathematics Subject Classification. Primary 43A46; Secondary 43A15, 43A25.

(C)1999 American Mathematical Society 
There are a number of well-known functional-analytic properties of a set which are equivalent to the definition of a $\Lambda(p)$ set (see [10, 37.9] and [12, 5.3] ). Since the Lorentz spaces are generalizations of the $L^{p}$ spaces, it is not surprising that similar characterizations of $\Lambda(p, q)$ sets may be obtained by straightforward modifications of known results for $\Lambda(p)$ sets. An easy, yet important, theorem states that if $p>2$, then the class of $\Lambda(p)$ sets is closed under the formation of finite unions. We will prove a similar result for $\Lambda(p, q)$ sets and discuss some related questions concerning unions. In [1], [3], [7], and [8] one finds a number of results on arithmetic and general set-theoretic properties of $\Lambda(p)$ sets. These properties deal with the structural nature of $\Lambda(p)$ sets. We will establish analogous properties for $\Lambda(p, q)$ sets.

\section{Preliminaries}

Let $\lambda$ denote the normalized Haar measure on $G$ and let $\|\cdot\|_{p}$ denote the usual $p$-norm where $p \in[1, \infty]$. Let $T$ denote the set of trigonometric polynomials on $G$ and $M$ denote the set of complex bounded Borel measures on $G$. For the reader's convenience, we shall give the definition and state some basic properties of Lorentz spaces; further details on these spaces are found in [6], [11], and [13].

Let $f$ be a complex-valued measurable function on $G$ which is finite almost everywhere. The distribution function $\lambda_{f}$ of $f$ is defined by

$$
\lambda_{f}(y)=\lambda\{x \in G:|f(x)|>y\} \text { for } y \geq 0 .
$$

The non-increasing rearrangement of $f$ is the function

$$
f^{*}(t)=\inf \left\{y>0: \lambda_{f}(y) \leq t\right\} \text { for } t \geq 0 .
$$

The Lorentz space $L(p, q)$ is defined as the set of equivalence classes of functions $f$ such that $\|f\|_{p, q}^{*}<\infty$, where

$$
\|f\|_{p, q}^{*}= \begin{cases}\left(\frac{q}{p} \int_{0}^{1}\left[t^{1 / p} f^{*}(t)\right]^{q} \frac{d t}{t}\right)^{1 / q} & \text { if } 1 \leq p, q<\infty \\ \sup _{t \in(0, \infty)} t^{1 / p} f^{*}(t) & \text { if } 1 \leq p \leq \infty, q=\infty .\end{cases}
$$

Since $\lambda_{f^{*}}=\lambda_{f}$, it follows that $\|f\|_{p, p}^{*}=\|f\|_{p}$ and hence $L(p, p)=L^{p}$ for all $p \in[1, \infty]$. The function $f \mapsto\|f\|_{p, q}^{*}$ is a quasi-norm for $L(p, q)$, but is not generally a norm. However, $L(p, q)$ does have a norm which is related to $\|\cdot\|_{p, q}^{*}$. To define this norm, consider a function $f$ and its averaging function $f^{* *}$ where

$$
f^{* *}(t)=\frac{1}{t} \int_{0}^{t} f^{*}(s) d s \text { for } t>0
$$

Then $L(p, q)$ can be taken as the set of equivalence classes of functions $f$ such that $\|f\|_{(p, q)}<\infty$, where

$$
\|f\|_{(p, q)}= \begin{cases}\left(\int_{0}^{\infty}\left[t^{1 / p} f^{* *}(t)\right]^{q} \frac{d t}{t}\right)^{1 / q} & \text { if } 1 \leq p, q<\infty \\ \sup _{t \in(0, \infty)} t^{1 / p} f^{* *}(t) & \text { if } 1 \leq p \leq \infty, q=\infty\end{cases}
$$


If $p=q \in\{1, \infty\}$ or if $p \in(1, \infty)$ and $q \in[1, \infty)$, then $L(p, q)$ is a Banach space with norm $\|\cdot\|_{(p, q)}$. The quasi-norm and norm are related by the inequality

$$
\left(\frac{p}{q}\right)^{1 / q}\|f\|_{p, q}^{*} \leq\|f\|_{(p, q)} \leq p^{\prime}\left(\frac{p}{q}\right)^{1 / q}\|f\|_{p, q}^{*}
$$

where $p \in(1, \infty)$ and $q \in[1, \infty]$. Note that $p^{\prime}$ is the index conjugate to $p$ and $\left(\frac{p}{q}\right)^{1 / q}=1$ if $q=\infty$. The most useful inequalities for the quasi-norm and norm are as follows. For $p \in[1, \infty]$ and $1 \leq q_{1}<q_{2} \leq \infty$,

$$
\|f\|_{p, q_{2}}^{*} \leq\|f\|_{p, q_{1}}^{*}
$$

and, for $p \in(1, \infty)$,

$$
\|f\|_{\left(p, q_{2}\right)} \leq\left(\frac{q_{1}}{p}\right)^{\left(q_{1}^{-1}-q_{2}^{-1}\right)}\|f\|_{\left(p, q_{1}\right)} .
$$

If $1<p_{1}<p_{2}<\infty$ and $q \in[1, \infty)$, then

$$
\|f\|_{p_{1}, q}^{*} \leq\left(\frac{p_{2}}{p_{2}-p_{1}}\right)^{1 / q}\|f\|_{p_{2}, \infty}^{*}
$$

A consequence of this last inequality is the proper inclusion

$$
L\left(p_{2}, q_{2}\right) \subset L\left(p_{1}, q_{1}\right)
$$

whenever $1<p_{1}<p_{2}<\infty$ and $q_{1}, q_{2} \in[1, \infty]$. As in [6, p. 368] define a total ordering on the set $J=(1, \infty) \times[1, \infty)$ by $(r, s)>(p, q)$ if and only if either $r>p$ or $r=p$ and $s<q$. Using this ordering and the inclusions in (1) and (6), it follows that

$$
L(r, s) \subset L(p, q) \text { if and only if }(r, s)>(p, q) .
$$

\section{BASIC PROPERTIES OF $\Lambda(p, q)$ SETS}

If $E$ is a finite subset of $\Gamma$, then $E$ is a $\Lambda(p, q)$ set for all $(p, q) \in J$ since $L(p, q)_{E}=L_{E}^{1}=T_{E}$. In contrast, the group $\Gamma$ itself is not a $\Lambda(p, q)$ set for any $(p, q) \in J$ since (1) shows that each such space $L(p, q)$ is always a proper subset of $L^{1}$. Our first results give simple relationships between $\Lambda(p, q)$ sets, $\Lambda(p)$ sets, and Sidon sets.

Theorem 3.1. Let $(p, q),(r, s) \in J$ and let $E \subset \Gamma$.

(a) If $E$ is a $\Lambda(p, q)$ set, then $E$ is a $\Lambda(r, s)$ set whenever $(p, q)>(r, s)$.

(b) If $E$ is a $\Lambda(p)$ set, then $E$ is a $\Lambda(r, s)$ set whenever $(p, p)>(r, s)$.

(c) If $E$ is a $\Lambda(p, q)$ set, then $E$ is a $\Lambda(r)$ set whenever $(p, q)>(r, r)$.

Proof. This is evident from (7) and the definition of $\Lambda(p)$ sets and $\Lambda(p, q)$ sets.

Theorem 3.2. Each Sidon set is a $\Lambda(p, q)$ set for all $(p, q) \in J$.

Proof. If $E$ is a Sidon set, then $[10,37.10]$ shows that $E$ is a $\Lambda(r)$ set for each $r \in(1, \infty)$. Given $(p, q) \in J$, if $r>p$, then $(r, r)>(p, q)$ and thus $E$ is a $\Lambda(p, q)$ set by Theorem 3.1(b).

Corollary 3.3. Each infinite subset of $\Gamma$ contains an infinite set which is a $\Lambda(p, q)$ set for every $(p, q) \in J$. 
Proof. By $[10,37.18]$ each infinite subset of $\Gamma$ contains an infinite Sidon set; hence the result follows from Theorem 3.2.

Theorem 3.2 and Corollary 3.3 are generalizations of classical results about Sidon sets and $\Lambda(p)$ sets. The following result is also such a generalization.

Theorem 3.4. There exists a subset of $\Gamma$ which is a $\Lambda(p, q)$ set for all $(p, q) \in J$ but is not a Sidon set.

Proof. By $[12,5.14] \Gamma$ contains an infinite subset $E$ which is a $\Lambda(r)$ set for all $r \in(1, \infty)$ but is not a Sidon set. If $(p, q) \in J$, then $(r, r)>(p, q)$ whenever $r>p$, and thus $E$ is a $\Lambda(p, q)$ set by Theorem 3.1(b).

In [6, Section 3] the following pair of lacunary sets based on Lorentz spaces are introduced.

Definition 3.5. Let $E \subset \Gamma$ and let $(p, q) \in J$.

(a) The set $E$ is a $\Lambda_{1}(p, q)$ set if there exists $s \in(1, p)$ such that $L(p, q)_{E}=$ $L(s, q)_{E}$.

(b) The set $E$ is a $\Lambda_{2}(p, q)$ set if there exists $r \in(q, \infty)$ such that $L(p, q)_{E}=$ $L(p, r)_{E}$.

As shown for $\Lambda(p, q)$ sets, each finite subset of $\Gamma$ is a $\Lambda_{1}(p, q)$ set and a $\Lambda_{2}(p, q)$ set for every $(p, q) \in J$. As well, $\Gamma$ itself is not a $\Lambda_{1}(p, q)$ set or a $\Lambda_{2}(p, q)$ set for any $(p, q) \in J$. The $\Lambda_{2}(p, q)$ sets are used in [6] to establish a characterization theorem for Lorentz-improving measures [6,3.4]. Some inclusions between $\Lambda_{2}(p, q)$ sets, $\Lambda_{1}(p, q)$ sets, and $\Lambda(p, q)$ sets are given in the following result.

Theorem 3.6. Let $E \subset \Gamma$ and let $(p, q) \in J$.

(a) If $E$ is a $\Lambda_{1}(p, q)$ set, then $E$ is a $\Lambda(r, s)$ set whenever $(r, s)<(u, u)$ and $1<u<p$.

(b) If $E$ is a $\Lambda(p, q)$ set, then $E$ is a $\Lambda_{1}(r, s)$ set whenever $(p, q) \geq(r, s)$.

(c) If $E$ is a $\Lambda(p, q)$ set, then $E$ is a $\Lambda_{2}(r, s)$ set whenever $(p, q) \geq(r, s)$.

Proof. (a) If $E$ is a $\Lambda_{1}(p, q)$ set, then [6,3.1(b)] shows that $E$ is a $\Lambda(u)$ set for every $u \in(1, p)$. By Theorem 3.1(b) $E$ is also a $\Lambda(r, s)$ set whenever $(r, s)<(u, u)$.

(b) Assume $E$ is a $\Lambda(p, q)$ set and let $(p, q) \geq(r, s)$. Then for each $w \in(1, r)$,

$$
L_{E}^{1} \subseteq L(p, q)_{E} \subseteq L(r, s)_{E} \subseteq L(w, s)_{E} \subseteq L_{E}^{1}
$$

and thus $E$ is a $\Lambda_{1}(r, s)$ set.

(c) If $E$ is a $\Lambda(p, q)$ set and $(p, q) \geq(r, s)$, then

$$
L_{E}^{1} \subseteq L(p, q)_{E} \subseteq L(r, s)_{E} \subseteq L(r, w)_{E} \subseteq L_{E}^{1}
$$

for each $w \in(s, \infty)$. This shows $E$ is a $\Lambda_{2}(r, s)$ set.

\section{Equivalent Statements For $\Lambda(p, q)$ Sets}

There are a number of functional-analytic properties of a subset $E$ of $\Gamma$ which are equivalent to the definition of a $\Lambda(p, q)$ set. These properties are analogous to some well-known and useful statements about a set which are equivalent to the definition of a $\Lambda(p)$ set (see [10, 37.7 and 37.9] and [12, 5.3]). The first of two results gives a characterization of $\Lambda(p, q)$ sets in terms of norms and quasi-norms, and is almost a full generalization of $[10,37.7]$. 
Theorem 4.1. Let $E \subset \Gamma$, let $(p, q),(r, s) \in J$, and assume $r>p$. The following assertions are equivalent:

(i) $E$ is a $\Lambda(r, s)$ set;

(ii) $L(r, s)_{E}=L(p, q)_{E}$;

(iii) there exists a constant $k$ such that $\|f\|_{r, s}^{*} \leq k\|f\|_{p, q}^{*}$ for every $f \in T_{E}$;

(iv) there exists a constant $k$ such that $\|f\|_{r, s}^{*} \leq k\|f\|_{1}$ for every $f \in T_{E}$;

(v) $M_{E}=L(r, s)_{E}$.

Proof. We will just outline the proof of each implication; the reader who is interested in the details of the original proof for $\Lambda(p)$ sets can refer to [10, 37.7].

(i $\Rightarrow$ ii) If $E$ is a $\Lambda(r, s)$ set where $r>p$, then $(r, s)>(p, q)$ and hence $L(r, s)_{E}=L_{E}^{1}$ and $L(r, s)_{E} \subseteq L(p, q)_{E}$ by (7). Since $L(p, q)_{E} \subseteq L_{E}^{1}$, assertion (ii) follows trivially.

(ii $\Rightarrow$ iii) If $L(r, s)_{E}=L(p, q)_{E}$ where $r>p$, then $(r, s)>(p, q)$ and thus the mapping $f \mapsto f$ of $L(r, s)_{E}$ into $L(p, q)_{E}$ is surjective and continuous. Since $L(r, s)_{E}$ and $L(p, q)_{E}$ are Banach spaces [5, 14.2], it follows from the open mapping theorem that there exists a constant $k$ such that

$$
\|f\|_{(r, s)} \leq k\|f\|_{(p, q)} \text { for all } f \in L(p, q)_{E} .
$$

Assertion (iii) is now clear as a result of this inequality, (2), and the fact that $T_{E} \subseteq L(p, q)_{E}$.

(iii $\Rightarrow$ iv) Since $L(r, s)_{E} \subseteq L(p, q)_{E}$ and $T_{E}$ is dense in both of these spaces, it follows from (iii) that $L(r, s)_{E}=L(p, q)_{E}$. Let $p_{1}$ and $p_{2}$ satisfy $p<p_{1}<p_{2}<r$. Then $L(r, s)_{E} \subseteq L_{E}^{p_{2}} \subseteq L_{E}^{p_{1}} \subseteq L(p, q)_{E}$ and hence $L_{E}^{p_{2}}=L_{E}^{p_{1}}$. By $[10,37.7]$ $L_{E}^{p_{2}}=L_{E}^{1}$ and thus $L(r, s)_{E}=L(p, q)_{E}=L_{E}^{1}$. By the open mapping theorem there exists a constant $k$ such that $\|f\|_{(r, s)} \leq k\|f\|_{1}$ for all $f \in L_{E}^{1}$. Combining this inequality with those in (2) and assertion (iii) yields (iv).

(iv $\Rightarrow \mathrm{v}$ ) From (1) and the Radon-Nikodym theorem, $L(r, s)_{E}$ may be regarded as a subset of $M_{E}$. We will show that $M_{E} \subseteq L(r, s)_{E}$. Let $\mu \in M_{E}$ and let $h \in T$. Then $\mu * h \in T_{E}$ and from (iv),

$$
\|\mu * h\|_{r, s}^{*} \leq k\|\mu * h\|_{1} \leq k\|\mu\|\|h\|_{1} .
$$

It follows from this inequality and (2) that

$$
\sup \left\{\|\mu * h\|_{(r, s)}: h \in T,\|h\|_{1} \leq 1\right\}<\infty .
$$

A straightforward modification of $[10,35.11]$ shows there exists a function $g \in$ $L(r, s)$ such that $d \mu=g d \lambda$. Since $\hat{\mu}$ vanishes off of $E, \hat{g}$ does also and thus $g \in L(r, s)_{E}$. This proves $\mu \in L(r, s)_{E}$ which establishes (v).

$(\mathrm{v} \Rightarrow \mathrm{i}) \quad$ If $L(r, s)_{E}=M_{E}$, then $L_{E}^{1} \subseteq M_{E}=L(r, s)_{E} \subseteq L_{E}^{1}$ and hence $L(r, s)_{E}=L_{E}^{1}$ which gives (i).

The proof of Theorem 4.1 shows that assertions (i), (iv), and (v) there are equivalent under the weaker hypothesis $(r, s)>(p, q)$. However, the full equivalence of assertions (i)-(v) has not been established for pairs $(r, s),(p, q)$ where $p=r$ and $q<s$. It is to this extent that Theorem 4.1 is not a complete generalization of $[10$, 37.7].

The next result gives additional properties of a set which are equivalent to the definition of a $\Lambda(p, q)$ set. These properties complement those in Theorem 4.1 in that they characterize $\Lambda(p, q)$ sets in terms of the dual of a Lorentz space. This result is almost a full generalization of the equivalences for $\Lambda(p)$ sets as found in $[10,37.9]$. 
Theorem 4.2. Let $E \subset \Gamma$ and let $(p, q) \in J$. The following assertions are equivalent:

(i) $\quad E$ is a $\Lambda(p, q)$ set;

(ii) for each $g \in L\left(p^{\prime}, q^{\prime}\right)$ there exists an $h \in L^{\infty}$ such that $\hat{g}(\gamma)=\hat{h}(\gamma)$ for all $\gamma \in E$;

(iii) for each $g \in L\left(p^{\prime}, q^{\prime}\right)$ there exists a continuous function $h$ such that $\hat{g}(\gamma)=$ $\hat{h}(\gamma)$ for all $\gamma \in E$.

If $p>2$, then statements (i)-(iii) above are equivalent to the assertion:

(iv) for each $g \in L\left(p^{\prime}, q^{\prime}\right), \sum_{\gamma \in E}|\hat{g}(\gamma)|^{2}<\infty$.

Proof. Again we will just outline the proof of each implication and refer the interested reader to $[10,37.9]$ for the original details concerning $\Lambda(p)$ sets.

$(\mathrm{i} \Rightarrow \mathrm{ii}) \quad$ This follows very closely the proof that (i) implies (ii) in $[10,37.9]$ except that Hölder's inequality for Lorentz spaces $[13,3.5]$ is used in place of the standard Hölder inequality.

(ii $\Rightarrow$ iii) Assume (ii) and let $g \in L\left(p^{\prime}, q^{\prime}\right)$. A factorization theorem for Lorentz spaces $([5,14.3]$ and $[10,32.33(\mathrm{~d})])$ shows there exist an $f \in L^{1}$ and a $g_{1} \in L\left(p^{\prime}, q^{\prime}\right)$ such that $g=f * g_{1}$. Now (ii) implies there is an $h_{1} \in L^{\infty}$ such that $\hat{h_{1}}(\gamma)=\hat{g_{1}}(\gamma)$ for all $\gamma \in E$. Let $h=f * h_{1}$ and note from [9, 20.16] that $h$ is continuous. One easily checks that $\hat{g}(\gamma)=\hat{h}(\gamma)$ for all $\gamma \in E$ and this establishes (iii).

(iii $\Rightarrow$ i) The proof of this implication follows very closely the proof that (iii $\Rightarrow$ i) in $[10,37.9]$. For convenience and completeness we outline the argument here for Lorentz spaces. Using $[10,35.7(\mathrm{c})]$ it is straightforward to verify that $L_{E^{c}}\left(p^{\prime}, q^{\prime}\right)$ is a closed two-sided ideal in $L\left(p^{\prime}, q^{\prime}\right)$ where $E^{c}$ denotes the complement of $E$ in $\Gamma$. As well, one checks that for a function $g \in L\left(p^{\prime}, q^{\prime}\right), g+L_{E^{c}}\left(p^{\prime}, q^{\prime}\right)=$ $h+L_{E^{c}}\left(p^{\prime}, q^{\prime}\right)$ precisely when $\hat{g}(\gamma)=\hat{h}(\gamma)$ for all $\gamma \in E$. There exists a constant $k$ such that, for each $g \in L\left(p^{\prime}, q^{\prime}\right)$, there is some continuous function $h$ which satisfies $h+L_{E^{c}}\left(p^{\prime}, q^{\prime}\right)=g+L_{E^{c}}\left(p^{\prime}, q^{\prime}\right)$ and $\|h\|_{u} \leq k\|g\|_{\left(p^{\prime}, q^{\prime}\right)}$. Here $\|\cdot\|_{u}$ denotes the uniform norm. Now let $f \in T_{E}$, let $g \in L\left(p^{\prime}, q^{\prime}\right)$, and consider the function $h$ above. Since $\hat{h}(\gamma)=\hat{g}(\gamma)$ for all $\gamma \in E$, it follows from [10, 34.33(ii)] that

$$
\int_{G} \bar{f}(s)(g(s)-h(s)) d \lambda(s)=0 .
$$

We see that

$$
\left|\int_{G} \bar{f}(s) g(s) d \lambda(s)\right| \leq\|h\|_{u}\|f\|_{1} \leq k\|g\|_{\left(p^{\prime}, q^{\prime}\right)}\|f\|_{1} .
$$

From this inequality and the duality result in $[2,(2.5)$, p. 9], it follows that $\|f\|_{(p, q)} \leq k\|f\|_{1}$. Assertion (i) is now evident from (2) and Theorem 4.1.

For the final implication assume that $p>2$.

(iii $\Rightarrow$ iv) If $g \in L\left(p^{\prime}, q^{\prime}\right)$, consider a continuous function $h$ such that $\hat{g}(\gamma)=\hat{h}(\gamma)$ for all $\gamma \in E$. Then $\sum_{\gamma \in E}|\hat{g}(\gamma)|^{2}=\sum_{\gamma \in E}|\hat{h}(\gamma)|^{2} \leq\|h\|_{2}^{2}<\infty$ which yields (iv).

(iv $\Rightarrow$ i) Following $[10,37.9]$ suppose that $\sum_{\gamma \in E}|\hat{g}(\gamma)|^{2}<\infty$ for each $g \in$ $L\left(p^{\prime}, q^{\prime}\right)$. Since $p>2,(1)$ shows that $L^{2} \subset L\left(p^{\prime}, q^{\prime}\right)$ and thus the mapping $f \mapsto f$ from $L^{2}$ into $L\left(p^{\prime}, q^{\prime}\right)$ is continuous. By $[10,28.43]$ there exists an $h \in L^{2}$ such that $\hat{h}(\gamma)=\hat{g}(\gamma)$ for all $\gamma \in E$ and $\hat{h}$ vanishes off of $E$. It follows from the proof of (iii $\Rightarrow \mathrm{i})$ and $(2)$ that there exists a constant $k$ such that $\|f\|_{p, q}^{*} \leq k\|f\|_{2}$ for all $f \in T_{E}$. Theorem 4.1 now shows that $E$ is a $\Lambda(p, q)$ set which establishes (i). 
Theorem 4.2 is not a complete generalization of $[10,37.9]$ as the equivalence of assertions (i)-(iv) has not been established for $(p, q)$ where $p=2$ and $q<2$.

\section{Set-theoretic AND ARITHMetic PRoperties}

An important problem in the theory of lacunary sets is the so-called "union problem". The problem is to determine whether a particular type of lacunary set is closed under finite unions. The following instances of this problem are well-known. If $p>2$ and if $E_{1}$ and $E_{2}$ are $\Lambda(p)$ sets, then $E_{1} \cup E_{2}$ is also a $\Lambda(p)$ set [10,37.21]. If $p \in(1, \infty)$, then $E_{1} \cup E_{2}$ is a $\Lambda(p)$ set whenever $E_{1}$ is a $\Lambda(p)$ set consisting of non-negative integers and $E_{2}$ is a $\Lambda(p)$ set consisting of negative integers [14, 4.4]. Lastly, if $E_{1}$ and $E_{2}$ are Sidon sets, then so is $E_{1} \cup E_{2}$ [12, 3.5]. These three examples motivate the following results for $\Lambda(p, q)$ sets.

Theorem 5.1. Let $(p, q) \in J$ and assume $p>2$. If $E_{1}$ and $E_{2}$ are $\Lambda(p, q)$ sets, then $E_{1} \cup E_{2}$ is a $\Lambda(p, q)$ set.

Proof. The argument is similar to that for $\Lambda(p)$ sets found in [10, 37.21]. Assume $E_{1}$ and $E_{2}$ are $\Lambda(p, q)$ sets and let $E=E_{1} \cup E_{2}$; we may assume the union is disjoint. By Theorem 4.1 and (2) there exist constants $k_{j}, j=1,2$, such that $\|f\|_{(p, q)} \leq k_{j}\|f\|_{2}$ for each $f \in T_{E_{j}}$. Let $k=\max \left\{k_{1}, k_{2}\right\}$ and let $f \in T_{E}$. Since $f$ can be written $f=f_{1}+f_{2}$ where $f_{j} \in T_{E_{j}}$, it follows from the PeterWeyl theorem that $\|f\|_{2}^{2}=\left\|f_{1}\right\|_{2}^{2}+\left\|f_{2}\right\|_{2}^{2}$ and thus $\left\|f_{j}\right\|_{2} \leq\|f\|_{2}$. Now $\|f\|_{(p, q)} \leq$ $\left\|f_{1}\right\|_{(p, q)}+\left\|f_{2}\right\|_{(p, q)} \leq 2 k\|f\|_{2}$, hence Theorem 4.1 and (2) imply that $E$ is a $\Lambda(p, q)$ set.

An obvious consequence of Theorem 5.1 is that a finite union of $\Lambda(p, q)$ sets is itself a $\Lambda(p, q)$ set. However, this is a best possible result in the sense that one cannot generally replace the word "finite" with "infinite" as $\Gamma$ is not a $\Lambda(p, q)$ set for any $(p, q) \in J$. Our next result is a generalization of $[14,4.4]$ where the analysis takes place on the circle group and its dual, the integers.

Theorem 5.2. Let $E_{1}$ be a set of non-negative integers and let $E_{2}$ be a set of negative integers. If $E_{1}$ and $E_{2}$ are $\Lambda(p, q)$ sets for some $(p, q) \in J$, then the set $E=E_{1} \cup E_{2}$ is also a $\Lambda(p, q)$ set.

Proof. Choose $(r, s) \in J$ with $p>r$ and hence $(p, q)>(r, s)$. By Theorem 3.1(a) both $E_{1}$ and $E_{2}$ are $\Lambda(r, s)$ sets. It follows from Theorem 4.1 and (2) that there are constants $k_{j}, j=1,2$, with $\left\|f_{j}\right\|_{(p, q)} \leq k_{j}\left\|f_{j}\right\|_{(r, s)}$ for all $f_{j} \in T_{E_{j}}$. Let $f \in T_{E}$ and write $f=f_{1}+f_{2}$ where $f_{j} \in T_{E_{j}}$. If $1<r_{1}<r<r_{2}<\infty$, then $L^{r_{2}} \subseteq L(r, s) \subseteq L^{r_{1}}$, and thus the M. Riesz theorem [4, 12.9.1] shows there are constants $c_{1}$ and $c_{2}$ such that $\left\|f_{j}\right\|_{r_{m}} \leq c_{m}\|f\|_{r_{m}}$ where $j, m=1,2$. Interpolate via [11, p. 264] to conclude there exists a constant $k$ such that $\left\|f_{j}\right\|_{(r, s)} \leq k\|f\|_{(r, s)}$. We see that

$$
\|f\|_{(p, q)} \leq\left(k_{1}+k_{2}\right)\left\|f_{j}\right\|_{(r, s)} \leq\left(k_{1}+k_{2}\right)\|f\|_{(r, s)} .
$$

Since $p>r$, it follows from Theorem 4.1 that $E$ is a $\Lambda(p, q)$ set.

If $E \subseteq \Gamma$ and $\tau \in \Gamma$, the translate of $E$ by $\tau$ is the set $\tau E=\{\tau \gamma: \gamma \in E\}$. Our next result shows that $\Lambda(p, q)$ sets are translation-invariant.

Theorem 5.3. If $E$ is a $\Lambda(p, q)$ set for some $(p, q) \in J$, then $\tau E$ is a $\Lambda(p, q)$ set for each $\tau \in \Gamma$. 
Proof. By (1) we need only verify the containment $L_{\tau E}^{1} \subseteq L(p, q)_{\tau E}$. If $f \in L_{\tau E}^{1}$ and $\gamma \in \Gamma$, then

$$
\begin{aligned}
\left(\tau^{-1} f\right)^{\wedge}(\gamma) & =\int_{G} \tau^{-1}(s) f(s) \bar{\gamma}(s) d \lambda(s) \\
& =\int_{G} f(s) \overline{(\tau \gamma)}(s) d \lambda(s)=\hat{f}(\tau \gamma) .
\end{aligned}
$$

If $\gamma \notin E$, then $\tau \gamma \notin \tau E$ and thus $\left(\tau^{-1} f\right)^{\wedge}$ vanishes off of $E$. Since $f$ and $\tau^{-1} f$ have equal distribution functions, we see that $\tau^{-1} f \in L_{E}^{1}$ and, as $E$ is a $\Lambda(p, q)$ set, $f \in L(p, q)_{\tau E}$.

If $E$ is a $\Lambda(p, q)$ set and $F$ is a finite subset of $\Gamma$, then $F E=\{\tau \gamma: \tau \in F, \gamma \in E\}$ is also a $\Lambda(p, q)$ set. As mentioned above, one cannot replace $F$ with an arbitrary infinite subset of $\Gamma$ as $\Gamma$ is not a $\Lambda(p, q)$ set.

We now consider arithmetic and geometric properties of $\Lambda(p, q)$ sets. It is known that Sidon sets and $\Lambda(p)$ sets for $p>2$ do not contain large parts of certain sets that are themselves generalized arithmetic progressions [12, Chapter 6]. This result for $\Lambda(p)$ sets was generalized and extended for all $p>1$ in [8].

Definition 5.4 ([8, 1.1 and 1.2]). A subset $P$ of $\Gamma$ is called a pseudo-parallelepiped of dimension $N$ if $P=\prod_{i=1}^{N}\left\{\gamma_{i}, \tau_{i}\right\}$ where $\gamma_{i}, \tau_{i} \in \Gamma, 1 \leq i \leq N$. A parallelepiped $P$ of dimension $N$ is a pseudo-parallelepiped of dimension $N$ such that $P$ consists of $2^{N}$ elements.

As remarked in [8, p. 144], pseudo-parallelepipeds are generalizations of arithmetic progressions of integers. Our first result is an easy generalization of $[8,1.2]$.

Theorem 5.5. If $E$ is a $\Lambda(p, q)$ set for some $(p, q) \in J$, then there exists an integer $N$ such that $E$ does not contain any parallelepipeds of dimension $N$.

Proof. This follows immediately from $[8,1.2]$ by noting from Theorem $3.1(\mathrm{c})$ that $E$ is a $\Lambda(r)$ set for each $r \in(1, p)$.

The next theorem extends the result of $[8,2.4]$ which gives an upper bound on the cardinality of the intersection of a $\Lambda(p)$ set and a generalized arithmetic progression. If $F$ is a finite subset of $\Gamma$, let $|F|$ denote the cardinality of $F$.

Theorem 5.6. Let $E$ be a $\Lambda(p, q)$ set for some $(p, q) \in J$. There exist constants $c>0$ and $\epsilon \in(0,1)$ such that if $S$ is an arithmetic progression in $\Gamma$ of length $N$, then $|E \cap S| \leq c N^{\epsilon}$.

Proof. This follows easily from $[8,2.3$ and 2.4] since $E$ is a $\Lambda(r)$ set for each $r \in$ $(1, p)$.

A consequence of Theorem 5.6 is that, like $\Lambda(p)$ sets, a $\Lambda(p, q)$ set cannot contain a generalized arithmetic progression of arbitrary length. For $p \in(1,2]$ it is not known whether the union of two $\Lambda(p)$ sets is itself a $\Lambda(p)$ set. However $[8,2.11]$ shows that $E_{1} \cup E_{2}$ does not contain parallelepipeds of arbitrarily large dimension if $E_{1}$ and $E_{2}$ are $\Lambda(p)$ sets for $p>1$. It is not difficult to see that this result is also true for $\Lambda(p, q)$ sets for all $(p, q) \in J$. Further connections between parellelepipeds, $\Lambda(p)$ sets, and $\Lambda(p, q)$ sets are also seen in $[8,4.1]$.

There is an interesting dichotomy in regards to the question of whether one class of $\Lambda(p)$ sets is contained in another. If $p \in(1,2)$, then each $\Lambda(p)$ set is also a 
$\Lambda(p+\epsilon)$ set for some $\epsilon>0$ (see [1, Main Theorem] and [7, Main Theorem]). On the other hand, it is shown in [3, Theorem 2] that, for each $p>2$, there exists a $\Lambda(p)$ subset of the integers which is not a $\Lambda(r)$ set for any $r>p$. Both of these results have consequences in our study of $\Lambda(p, q)$ sets.

Theorem 5.7. If $E$ is a $\Lambda\left(p_{1}, q_{1}\right)$ set for some $\left(p_{1}, q_{1}\right) \in J$ and $p_{1} \in(1,2)$, then $E$ is a $\Lambda(p, q)$ set for some $(p, q)>\left(p_{1}, q_{1}\right)$.

Proof. Consider first the case where $q_{1} \in\left[1, p_{1}\right)$. By $(1), L\left(p_{1}, q_{1}\right) \subset L^{p_{1}}$ and thus $E$ is a $\Lambda\left(p_{1}\right)$ set. From [7, Main Theorem] $E$ is $\Lambda\left(p_{1}+\epsilon\right)$ set for some $\epsilon>0$ and hence the result follows easily by just letting $p=q=p_{1}+\epsilon$. Suppose now that $q_{1}>p_{1}$. By $(7), L\left(p_{1}, q_{1}\right) \subset L^{r}$ for all $r \in\left(1, p_{1}\right)$ and thus $E$ is a $\Lambda(r)$ set for each such $r$. We will show that $E$ is a $\Lambda(s)$ set for some $s>p_{1}$. The conclusion will then follow from the case above.

As motivated by [7], for $r \in\left(1, p_{1}\right]$ and $n \geq 2$, let $s$ be defined by the equation

$$
\frac{1}{s}=\frac{1}{r}+\left(\frac{2-r}{4 r}\right) c(n) \text { where } c(n)=\frac{\log \left(1-\frac{1}{n^{2}}\right)}{\log \left(2 n^{2}\right)} \text {. }
$$

Note that $s>r$ since $c(n)<0$. Rearrange (8) so that $s=s(r, n)=\frac{4 r}{4+(2-r) c(n)}$. By letting $k(r, r / 2 ; E)=\inf \left\{\|f\|_{r}: f \in T_{E},\|f\|_{r / 2} \leq 1\right\}$ we see from [7, Remark, p. 7] that for each $r \in\left(1, p_{1}\right)$, if $n \geq 4(k(r, r / 2 ; E))^{r}$, then $E$ is a $\Lambda(s)$ set. For $n \geq 2$, let $r_{n}=p_{1}-\frac{1}{2}\left[s\left(p_{1}, n\right)-p_{1}\right]$. It is clear that $r_{n}<p_{1}$. Since $c(n) \rightarrow 0^{-}$as $n \rightarrow \infty$, it follows that $r_{n}>1$ for all sufficiently large $n$. Consequently for these such $n, E$ is a $\Lambda(s)$ set where $s=s\left(r_{n}, n\right)$. A straightforward calculation shows that $s\left(r_{n}, n\right)>p_{1}$ if $n$ is sufficiently large. This completes the proof.

Theorem 5.8. For each $\left(p_{1}, q_{1}\right) \in J$ where $q_{1} \geq p_{1}>2$, there exists a $\Lambda\left(p_{1}, q_{1}\right)$ set in $\Gamma$ which is not a $\Lambda(p, q)$ set for any $(p, q) \in J$ such that $p>p_{1}$.

Proof. Let $\left(p_{1}, q_{1}\right) \in J$ where $q_{1} \geq p_{1}>2$. By [3, Theorem 1] there exists a subset $E$ of $\Gamma$ which is a $\Lambda\left(p_{1}\right)$ set but is not a $\Lambda(r)$ set for any $r>p_{1}$. It follows from (1) that $E$ is a $\Lambda\left(p_{1}, q_{1}\right)$ set and from (7) that $E$ is not a $\Lambda(p, q)$ set if $p>p_{1}$.

\section{REMARKS}

A pervasive theme in this paper has been to generalize results for $\Lambda(p)$ sets by expressing them as theorems about $\Lambda(p, q)$ sets. This theme has been developed by capitalizing on the structural similarities existing between $L^{p}$ spaces and $L(p, q)$ spaces. A similar idea is exploited in [5] and [6] where the theory of $L^{p}$-improving measures is extensively generalized and subsequently leads to the theory of Lorentzimproving measures.

Theorem 5.7 and the inclusions found in (1) suggest the following question. Suppose $E$ is a $\Lambda\left(2, q_{1}\right)$ set where $q_{1}>2$. Is it true that $E$ is also a $\Lambda(2, q)$ set for some $q \in\left[1, q_{1}\right)$ ? As mentioned just before Theorem 5.7, the analogous question for $\Lambda(p)$ sets has been answered affirmatively: if $p \in(1,2)$, then each $\Lambda(p)$ set is also a $\Lambda(p+\epsilon)$ set for some $\epsilon>0$ (see [1, Main Theorem] and [7, Main Theorem]). The techniques used in these two papers for the analysis of $\Lambda(p)$ sets do not yet appear to be modifiable so as to yield a definite answer to the above question for $\Lambda(p, q)$ sets. Theorem 5.7 is, however, a natural result in this direction. Note that if $E$ is a $\Lambda\left(2, q_{1}\right)$ set for some $q_{1}>2$, then $E$ is also a $\Lambda(p)$ set for all $p \in(1,2)$ since $L\left(2, q_{1}\right) \subset L^{p}$. Consequently, if there does exist such a set $E$ which is not a 
$\Lambda(2, q)$ set for any $q \in\left[1, q_{1}\right)$, then $E$ also has the interesting property that it is a $\Lambda(p)$ set for all $p \in(1,2)$ yet is not a $\Lambda(2)$ set. The reader is referred to [8] for some further issues regarding open questions on the relationship between $\Lambda(p)$ sets for $p \in(1,2)$, and $\Lambda(2)$ sets.

A major contribution to the theory of $\Lambda(p)$ sets is [3] where a solution to the celebrated $\Lambda(p)$ set problem is presented. The solution asserts that, for each $p>2$, there is a $\Lambda(p)$ set of integers which is not a $\Lambda(r)$ set for any $r>p$. The analogous problem for $\Lambda(p, q)$ sets is as follows. Does there exist a $\Lambda\left(p_{1}, q_{1}\right)$ set for some $\left(p_{1}, q_{1}\right)>(2,2)$ which is not a $\Lambda(p, q)$ set for any $(p, q)>\left(p_{1}, q_{1}\right)$ ? Theorem 5.8 is a minor result concerning this question. It is seen in [3] that the solution to the $\Lambda(p)$ set problem is obtained via intricate probabilistic methods. It is quite unclear as to whether these kinds of techniques can be modified so as to provide a solution to the problem for $\Lambda(p, q)$ sets.

A popular and successful direction for lacunary research is to generalize results for the integers and rephrase them in terms of the dual of a compact abelian group. A further degree of extension is attained when results for the dual of a compact abelian group can be abstracted in terms of the dual object of a compact group or hypergroup. There are a number of possibilities for further research suggested by these remarks and the results for $\Lambda(p, q)$ sets we have developed in this paper.

The author thanks the referee for his useful comments.

\section{REFERENCES}

[1] Bachelis, G. \& Ebenstein, S., On $\Lambda(p)$ sets, Pac. J. Math. 54(1) (1974), 35 - 38. MR 52:3887

[2] Bennett, C. \& Sharpley, R., Interpolation of operators, Academic Press, Orlando, 1988. MR 89e:46001

[3] Bourgain, J., Bounded orthogonal systems and the $\Lambda(p)$ set problem, Acta Math. 162(1989), 227-245. MR 90h:43008

[4] Edwards, R., Fourier Series, Vol. II, Springer-Verlag, New York, 1982. MR 83k:42001

[5] Grinnell, R., Lorentz-improving measures on compact abelian groups, Ph.D. Dissertation, Queen's University, 1991.

[6] Grinnell, R. \& Hare, K., Lorentz-improving measures, Ill. J. Math. Vol. 38, No. 3, (1994), 366-389. MR 95c: 43001

[7] Hare, K., An elementary proof on a result on $\Lambda(p)$ sets, Proc. Amer. Math. Soc. 104(3) (1988), 829 - 834. MR 89m:43012

[8] Hare, K., Arithmetic properties of thin sets, Pac. J. Math. 131(1) (1988), 143-155. MR 89a: 43010

[9] Hewitt, E. \& Ross, K., Abstract harmonic analysis, Vol. I, Springer-Verlag, Berlin, 1963. MR 28: 158

[10] Hewitt, E. \& Ross, K., Abstract harmonic analysis, Vol. II, Springer-Verlag, Berlin, 1970. MR 41:7378

[11] Hunt, R., On L $(p, q)$ spaces, Enseign. Math. 12 (1966), 249 - 276. MR 36:6921

[12] Lopez, J. \& Ross, K., Sidon sets, Marcel-Dekker, New York, 1975. MR 55:13173

[13] O'Neil, R., Convolution operators and L $(p, q)$ spaces, Duke Math. J. 30 (1969), 129-142. MR 26: 4193

[14] Rudin, W., Trigonometric series with gaps, Jour. Math. and Mech. 9(2) (1960), 203-227. MR 22:6972

[15] Yap, L., Some remarks on convolution operators and $L(p, q)$ spaces, Duke Math. J. (1969), 647-58. MR 40:3184

Department of Computer Science, Mathematics \& Physics, University of the West Indies, Cave Hill Campus, P.O. Box 64, Bridgetown, Barbados, West Indies

E-mail address: grinnell@uwichill.edu.bb 